\title{
Application of the Zero Suicide Model in Residency Training
}

\author{
Yael Holoshitz $^{1}$ (D) Beth Brodsky ${ }^{1} \cdot$ Sidney Zisook ${ }^{2} \cdot$ Joel Bernanke $^{3} \cdot$ Barbara Stanley $^{1}$ \\ Received: 6 June 2018 / Accepted: 8 January 2019 / Published online: 23 January 2019 \\ (C) Academic Psychiatry 2019
}

The increasing suicide rate in the USA is a national crisis. In 2016 , nearly 45,000 people in the USA died by suicide, which represents a $>30 \%$ increase between 2000 and 2016 [1]. Further, for every reported suicide, an estimated 25 suicide attempts occur. Suicide is the tenth leading cause of death in the USA, and psychiatrists are often on the frontlines of suicide prevention. Psychiatrists staff emergency departments (EDs) and serve on consultation-liaison services in general hospitals, evaluating suicide risk. In clinic settings, psychiatrists may function in clinical oversight roles for other mental health clinicians and be called upon to evaluate at-risk patients. Psychiatrists on inpatient units serve as primary providers and oversee discharge of at-risk patients. Additionally, with increased collaborative care models, psychiatrists play an increasingly important role in primary care settings [2]. To that end, it is critical that psychiatrists have the skills, knowledge, and clinical familiarity needed for suicide prevention.

In 2016, the Joint Commission ushered in what is hoped to become a new focus on suicide prevention, with the Sentinel Event Alert, which aims to "assist all health care organizations providing both inpatient and outpatient care to better identify and treat individuals with suicidal ideation" ([3], p. 1). There is increasing recognition of the need to train the next generation of medical professionals, including psychiatrists, in suicide prevention.

Concerted efforts to teach evidence-based approaches to suicide prevention in residency can provide guidance for more effective interventions. Psychiatrists and psychiatric residents should feel competent, if not comfortable, in identifying, assessing, and managing patients presenting with suicidal

Yael Holoshitz

Yael.holoshitz@nyspi.columbia.edu

1 New York State Psychiatric Institute, Columbia University College of Physicians and Surgeons, New York, NY, USA

2 University of California, San Diego, CA, USA

3 New York Presbyterian Hospital, Weill Cornell Medical Center/ Columbia University Medical Center, New York, NY, USA ideation at all levels of acuity and psychopathology. We suggest that adopting the Zero Suicide model of suicide prevention [4] in residency training may be helpful by providing an organized and integrated approach to working with suicidal patients. It is one approach to improving teaching in suicide prevention, and it has the potential to provide a comprehensive framework and to help consolidate residents' knowledge, skills, and attitudes about suicide prevention.

\section{The Zero Suicide Model}

The Zero Suicide Initiative is a strategic framework put forth by the National Strategy for Suicide Prevention and the National Action Alliance for Suicide Prevention [4]. It is a project of the Suicide Prevention Resource Center, supported by the Substance Abuse and Mental Health Services Administration [5]. The National Strategy for Suicide Prevention advocates a comprehensive approach to suicide prevention, including community, school, and primary care settings. The Zero Suicide Initiative focuses on one aspect of the National Strategy — health care systems - because suicidal patients frequently use health care services prior to their attempt or death $[6,7]$. The Zero Suicide Initiative was modeled, in part, after the "Perfect Depression Care" program of Michigan's Henry Ford Health System. Implementation of this program was correlated with a lowered rate of suicide in their patient population by $75 \%(p=0.007)$, from approximately 89 per 100,000 at baseline (in 2000) to approximately 22 per 100,000 for the 4-year follow-up period (2002-2005) $[8,9]$. Though causation cannot be assumed by these findings, these preliminary correlational findings are promising.

The guiding principle of the Zero Suicide Initiative is that for individuals being treated in behavioral health systems, death by suicide is preventable [4]. Though the "zero" in Zero Suicide may be unachievable and the goal itself may increase feelings of guilt and sense of failure for clinicians, as well as caregivers and survivors, when suicides occur [10], a contrasting view envisions the "zero" in Zero Suicide as an 
aspirational goal that projects a sense of hope and optimism about what mental health care can accomplish. This optimism can be seen as an antidote to the too-frequently felt experiences of demoralization and futility that may accompany work with high-risk patients.

Though the full details of the Zero Suicide Initiative are beyond the scope of this paper, the essence of the initiative is to implement concerted, systematic, structural, and cultural change in an ongoing way, by using up-to-date evidence and ongoing monitoring of quality [4]. The Zero Suicide Initiative aims to target suicide as an epidemic that can be treated and not only as a symptom of other psychiatric illness. The model contains seven essential components of comprehensive suicide-safer care, which encompass both implementation/ structural elements (leadership, training, improvement), and clinical elements (identify, engage, treat, transition) $[4,5]$.

The model highlights the necessity for leadership to demonstrate a commitment to safety and support for staff. Leadership should be committed to setting high standards of clinical care for suicidal patients, while at the same time maintaining a supportive, non-punitive attitude toward clinicians working with this high-risk population. The model emphasizes the need for ongoing training, particularly in brief, evidence-based, suicide prevention strategies designed to target suicide prevention directly. These suicide-specific interventions are applied alongside treatment of the underlying diagnosis.

Other important components of the model include implementation of a systematic suicide care protocol; suicide-safer care should be approached in the same systematic manner as treatment for conditions such as diabetes and high blood pressure. Systematic protocols should include elements such as ongoing screening, the collaborative Safety Planning Intervention (SPI) [11], access to specialty care, focus on reduction of lethal means, consistent engagement efforts, and monitoring during periods of high risk. Along these lines, the Zero Suicide model emphasizes the importance of provision of support during care transitions, such as discharge from the ED or hospital. Finally, the Zero Suicide model highlights the importance of continuous quality improvement and monitoring outcomes.

Elements of the Zero Suicide model have been adopted by the Veteran's Health Administration Hospital systems [12] and in other care settings. A recent quality improvement educational initiative at the Medical College of Wisconsin psychiatry residency program sought to incorporate elements of the Zero Suicide Initiative into clinical care, focusing on the inpatient population [12]. They increased use of safety plans from 10 to $52 \%$, focused on means restriction, conducted a postvention workshop focusing on emotional and professional aspects of losing a patient to suicide, and instituted quality care rounds (e.g., morbidity and mortality rounds). This initiative was met with enthusiasm, and residents actively engaged in an adoption of comprehensive suicide prevention, which resembled the Zero Suicide approach to patient safety [12]. Currently, researchers in New York State and Columbia University Department of Psychiatry are evaluating widespread implementation of Zero Suicide into outpatient behavioral health clinics serving $>80,000$ patients in a study funded by the National Institution of Mental Health [6].

\section{Current Residency Training in Suicide Prevention}

Current training in suicide prevention in US residency programs is varied and tends to focus on specific elements of suicide prevention, such as risk assessment, rather than an integrative approach. A survey of psychiatry chief residents indicated that while the vast majority of residency programs offer teaching on suicide assessment and care (including didactics and case conferences), residents expressed the need for more instruction on even the most common topics, such as identification of risk factors, warning signs, and standards of care [13]. Furthermore, teaching about suicide prevention in psychiatric residency tends to focus on treatment of underlying disorders linked with suicidal ideation (e.g., depression, psychosis). While disorder-specific treatment is vital, suicide-specific interventions may be indicated for suicidal patients. In many programs, the majority of teaching about suicide prevention may be in contexts not specifically dedicated to suicide prevention, such as in clinical case conferences, therapy supervision, and seminars on general topics in psychiatry $[14,15]$. To that end, it may be difficult for residents to integrate what they have learned about suicide prevention from multiple different settings and contexts into an overarching approach to working with individuals presenting with suicidal risk.

A review of the Accreditation Council for Graduate Medical Education (ACGME) Milestones Project for Psychiatric Residency Education highlights this gap in training [16]. Out of 22 identified milestones deemed as necessary for training, none specifically focuses on suicide prevention. Though several milestones address components necessary for suicide prevention, such as assessment of risk, determination of need for higher level of care, and management of patients in crisis, the absence of suicide prevention as an explicit core competency is notable.

The educational mission and philosophy set forth by the recent ACGME milestones project value systematic approaches to clinical care, as well as ongoing quality improvement, comprehensive services, and coordination of care with non-psychiatric providers. These domains emphasize a philosophy of training programs that will prepare the next generation of psychiatrists to be leaders in their field, and to that end, the systematic approach of Zero Suicide is a natural integration into core competency training. 


\section{Improving Residents' Training in the Care of Suicidal Patients}

Though the effectiveness of the application of the Zero Suicide model in residency training has not been studied, this perspective piece aims to demonstrate how the Zero Suicide model and its systematic approach to suicide prevention teaching could enhance residency training. One proposed way that such an approach could be implemented in residency training is via the AIM model: Assessment, Intervention, and Monitoring. This model was developed by Barbara Stanley and colleagues at the New York State Psychiatric Institute/ Columbia University Department of Psychiatry as a way to monitor and treat individuals at risk for suicide, using evidence-based best practices $[17,18]$. The AIM model embraces the clinical elements of the Zero Suicide model (i.e., identify, engage, treat, transition) and is one framework for applying the Zero Suicide model. A recent large-scale cohort comparison study demonstrated that use of the AIM model was associated with reduction in suicidal behavior and increased treatment engagement in suicidal patients 6-months after ED discharge [19].

The AIM model uses multiple evidence-based interventions for suicide prevention. In the Assessment portion of the AIM model, the clinician conducts a structured assessment of suicide risk using a comprehensive tool such as the Columbia-Suicide Severity Rating Scale (C-SSRS) [20]. Suicide prevention experts recommend universal screening with a validated instrument at regular intervals, though many individuals in health care settings do not receive such screening [21]. Suicidal ideation is quite common in many clinical populations; in a sample of adults with nonpsychotic major depressive disorder presenting for level 1 treatment in STAR $* \mathrm{D},>50 \%$ endorsed suicidal ideation at baseline [22]. Therefore, all adult patients in psychiatric settings should be screened for suicide, and clinicians should not wait until patients report suicidal ideation. Further, the field has shifted away from attempting to predict risk and toward preventing, or mitigating, risk factors [23]. As such, regular assessments should weigh distal and proximal risk and protective factors and monitor any changes in patients in an ongoing way, using this information to inform treatment planning $[6,23,24]$. This information gathering should be done mindfully, in the context of a connected, empathic clinical approach, in order to optimize efficacy of the structured tools.

In the Intervention portion of the AIM model, the clinician applies evidence-based brief interventions for suicide prevention, such as the SPI [11]. The SPI has been evaluated in a large-scale cohort comparison study of suicidal patients following ED discharge and found to reduce suicidal behavior and to increase treatment engagement in patients discharged from the emergency room for suicidal ideation [25]. Intervention also includes use of medications for treatment of underlying conditions and incorporation of family or other social supports into treatment planning. Restriction of lethal means, including limiting access to firearms, is also an important intervention that can reduce rates of suicide [26-29].

Finally, in the Monitoring portion of the AIM model, the clinician applies guidelines for ongoing monitoring and followup during high-risk periods such as during suicidal risk periods and transition in care. Multiple studies have indicated that contacting patients during high-risk periods, such as following discharge from an inpatient unit or from the $\mathrm{ED}$, can reduce suicide attempts and increase treatment engagement [30-32].

The AIM model can be applied to all care settings, and therefore, this approach can span all clinical experiences throughout training. This model can be applied and revisited in an ongoing manner throughout all clinical interactions. Approaching clinical care with the AIM model could thus be a helpful way for residents to conceptualize treatment of individuals who are at risk for suicide, and it could enrich residents' clinical acumen and ability to stratify risk and develop treatment plans.

For example, in an ED setting, residents can assess someone's suicide risk by using a structured suicide assessment tool, such as the C-SSRS. Weighing the proximal and distal risk and protective factors, the resident may determine that an individual is at elevated, but not imminent, risk and thus does not necessitate inpatient hospitalization. This same resident can conduct or oversee a safety plan with the patient prior to ED discharge and identify a solid disposition plan to ensure follow-up care is in place. The final step of the AIM model would be to implement structured follow-up and monitoring. The resident can work with the rest of the ED staff to discuss the option for one or more check-in calls to the patient following ED or inpatient discharge. The purpose of the calls is to assess for safety, discuss use of the SPI, and troubleshoot any potential barriers to connecting to the initial outpatient visit. This high-quality approach to clinical care applying the Zero Suicide-inspired AIM model could potentially enhance the quality of care that the patient receives in a busy ED setting. Of course, in some clinical contexts, follow-up and check-in calls may not be feasible, in which case, the resident can focus on assessment and intervention.

The AIM model could be applied in inpatient and outpatient settings as well. In the outpatient setting, a resident can use screening tools such as the C-SSRS for ongoing risk assessment during outpatient visits, develop a safety plan that is frequently revisited and revised, and conduct structured follow-up and monitoring check-in calls between visits during higher risk periods. This approach may decrease unnecessary hospitalizations or referral to the ED for outpatients who are presenting with increased suicidal ideation and provide a framework by which the resident can monitor clinical changes in higher risk patients in an ongoing manner. This structured, comprehensive approach to assessing and conceptualizing risk is likely to help mitigate the 
understandable anxiety that residents may experience in working with high-risk outpatients.

Application of the AIM model to residency training does not require any significant overhaul of didactics or clinical training. Many of these components are taught throughout residencyresidents working on inpatient units and in EDs learn about thresholds for admission and discharge, and residents working in outpatient settings work with higher risk patients. Residents learn risk factors and protective factors for suicide, including demographic and psychiatric factors. However, what is often missing is the synthesis and consolidation of these different components of suicide prevention, which may lead to gaps in knowledge and confusion about clinical management. Residents may find that they think differently about suicide prevention in the ED versus an outpatient setting. The AIM model has the potential to reduce this compartmentalized thinking, because its comprehensive nature spans various clinical contexts. The model highlights not only immediate assessment and intervention but ongoing monitoring and longer-term follow-up. Thus, residents on inpatient units or in EDs can be thinking about outpatient care for at risk individuals and vice versa, leading to an integration of clinical knowledge about suicide prevention and consolidation of skills.

One major challenge to the implementation of the Zero Suicide Initiative in residency training, through the AIM approach or other encompassing frameworks, may be in the structure of clinical teaching experiences. Clinicians who supervise residents may not be up-to-date on quickly evolving, state-of-theart suicide prevention practices. In some cases, such clinical teaching can perpetuate misinformation and myths about suicide prevention. For the proposed culture shift to occur, supervisors must also be educated, perhaps through in-services or mandatory continuing medical education trainings.

Along those lines, there may be practical limitations to implementing these changes. Some clinical services, such as the Psychiatric Emergency Department, are quite busy, and residents may need to quickly evaluate and triage patients. Residents may already feel overworked, and risk for burnout among medical trainees is high [33]. If not implemented properly, any change or addition to residency training may feel more like a burden than an educational opportunity. Care must be taken in introducing this new framework to residents and in supporting them in its use via leadership oversight and optimization of services, including team-based collaboration with other service staff such as triage and nursing. The workload need not fall solely on the physician, and in fact, collaboration is one key feature of Zero Suicide-informed frameworks. Further, it is possible to focus only on certain aspects of the AIM framework, such as structured risk assessment and the SPI. Ultimately, this approach could reduce residents' distress and increase their confidence in evaluating and treating highrisk patients in busy settings, as it strives to increase evidencebased approaches and improve system efficiency.
Another challenge in implementing the Zero Suicide Initiative in training is the nomenclature. As mentioned earlier, clinicians may be uncomfortable with perceived connotations of the word zero, feeling that it is an unreasonable goal. A recent qualitative study of 12 Swedish psychiatrists sought to understand how a similar model, Vision Zero for suicide, is perceived [34]. Though these psychiatrists pointed to many potential benefits of the initiative, including increased resources for suicide prevention, a positive signal from authorities, increased awareness in health care, and a "reaching for the stars" attitude, many expressed skepticism for the model. Among other concerns, psychiatrists interviewed felt that Vision Zero was unachievable, may increase physician anxiety, and may deter people from entering the field of psychiatry. These arguments have merit, and ample opportunity should be given to residents to discuss concerns they have about their work in suicide prevention. However, one stated mission of Zero Suicide is indeed to shift the onus away from the individual provider and onto the greater system and leadership. Its policies strive to create a transparent, supportive, and blamefree environment [6]. Regardless, the nomenclature should not be a major focus and certainly should not delay widespread implementation of this initiative.

Now is an opportune time to revisit suicide prevention in psychiatry and in residency training and to adjust clinical standards to reflect the progress, changing attitudes, and new practices for suicide prevention. In order to enhance clinical care, a culture shift must come from residency leadership and clinical directors to make suicide-specific care an ongoing and direct focus of treatment.

Funding Dr. Stanley received funding from the National Institute of Mental Health, Award Number 1R01MH112139.

\section{Compliance with Ethical Standards}

Disclosures Drs. Holoshitz, Brodsky, Zisook, and Bernanke have no conflicts of interest. Dr. Stanley reports grants from National Institute of Mental Health during the conduct of the study and royalties from the Research Foundation for Mental Hygiene, Inc., for commercial use of the Columbia-Suicide Severity Rating Scale (C-SSRS).

Publisher's Note Springer Nature remains neutral with regard to jurisdictional claims in published maps and institutional affiliations.

\section{References}

1. Center for Disease Control and Prevention (CDCP). 2016. Suicide injury deaths and rates per 100,000. CDCP Web-based Injury Statistics Query and Reporting System (WISQARS). [accessed 8 August 2018]. Available from https://www.cdc.gov/injury/wisqars/ fatal.html

2. Community Preventive Services Task Force. Recommendation from the community preventive services task force for use of collaborative care for the management of depressive disorders. Am J Prev Med. 2012;42:521-4. 
3. Joint Commission. Detecting and treating suicide ideation in all settings. Sentinel Event Alert [serial on the Internet] Oak Brook (IL): Joint Commission; 2016 Feb 24 [accessed 25 July 2017] (issue No. 56). Available from http://www.jointcommission.org/ assets/1/18/SEA 56 Suicide.pdf

4. Hogan MF, Grumet JG. Suicide prevention: an emerging priority for health care. Health Aff. 2016;35(6):1084-90.

5. Suicide Prevention Resource Center. Zero suicide in health and behavioral health care. 2017 [accessed 7 August 2017]; available from: http://zerosuicide.sprc.org

6. Labouliere CD, Vasan P, Kramer A, Brown G, Green K, Rahman $\mathrm{M}$, et al. "Zero suicide"- a model for reducing suicide in United States behavioral healthcare. Suicidologi. 2018;23(1):22-30.

7. Ahmedani BK, Simon GE, Stewart C, Beck A, Waitzfelder BE, Rossom R, et al. Health care contacts in the year before suicide death. J Gen Intern Med. 2014;29:870-7.

8. Coffey CE. Building a system of perfect depression care in behavioral health. Jt Comm J Qual Patient Saf. 2007;33(4):193-9.

9. Hampton T. Depression care effort brings dramatic drop in large HMO population's suicide rate. J Am Med Assoc. 2010;202(19): 1903-5.

10. Ehrlich MD. GAP committee on psychopathology. Envisioning zero suicide. Psychiatr Serv. 2016;67(3):255.

11. Stanley B, Brown GK. Safety planning intervention: a brief intervention to mitigate suicide risk. Cogn Behav Pract. 2012;19:25664.

12. Pheister M, Kangas G, Thompson C, Lehrmann J, Berger B, Kemp J. Suicide prevention and postvention resources: what psychiatry residencies can learn from the veteran's administration experience. Acad Psychiatry. 2014;28:600-4.

13. Melton BB, Coverdale JH. What do we teach psychiatric residents about suicide? A national survey of chief residents. Acad Psychiatry. 2009;33(1):47-50.

14. Ellis TE, Dickey TO, Jones EC. Patient suicide in psychiatry residency programs: a national survey of training and postvention practices. Acad Psychiatry. 1998;22(3):181-9.

15. Zisook S, Anzia J, Atri A, Baroni A, Clayton P, Haller E, et al. Teaching evidence-based approaches to suicide risk assessment and prevention that enhance psychiatric training. Compr Psychiatry. 2013;54:201-8.

16. Accreditation Council for Graduate Medical Education. The psychiatry milestone project [Accessed 7 Aug 2017] Available from http://www.acgme.org/What-We-Do/Accreditation/Milestones/ Milestones-by-Specialty

17. Stanley, B. Suicide: scope of the problem and strategies for prevention- the AIM Model. NYS-OMH Suicide Prevention Conference. Albany, NY, September 12, 2016. https:// nyssuicidepreventionconference.org

18. Brodsky BS, Spruch-Feiner A, Stanley B. The zero suicide model: applying evidence-based suicide prevention practices to clinical care. Front Psychiatry. 2018;9:33.

19. Stanley B, Brown GK, Brenner LA, Galfalvy HC, Currier GW, Knox KL, et al. Comparison of the safety planning intervention with follow-up vs. usual care of suicidal patients treated in the emergency department. JAMA Psychiatry. 2018;75(9):894-900.
20. Posner K, Brent D, Lucas C, Gould M, Stanley B, Brown G, et al. Columbia suicide severity rating scale. New York: Columbia University; 2008.

21. Posner K, Brown GK, Stanley B, Brent DA, Yershova KV, Oquendo MA, et al. The Columbia-Suicide Severity Rating Scale: initial validity and internal consistency findings from three multisite studies with adolescents and adults. Am J Psychiatry. 2011;168:1266-77.

22. Zisook S, Trivedi MH, Warden D, Lebowitz B, Thase ME, Stewart $\mathrm{JW}$, et al. Clinical correlates of the worsening or emergence of suicidal ideation during SSRI treatment of depression: an examination of citalopram in the STAR $* \mathrm{D}$ study. J Affect Disord. 2009; 117(1-2):63-73.

23. Pisani A, Murrie DC, Silverman MM. Reformulating suicide risk formulation: from prediction to prevention. Acad Psychiatry. 2016;40:623-9.

24. Jobes DA, Wong SA, Conrad AK, Drozd JF, Neal-Walden T. The collaborative assessment and management of suicidality versus treatment as usual: a retrospective study with suicidal outpatients. Suicide Life Threat Behav. 2005;35:483-97.

25. Mann HH Apter A, Bertolote J, Beautrais A, Currier D, Haas A, et al. Suicide prevention strategies: a systematic review. JAMA. 2005;294(16):2064-74.

26. Yip PS, Law CK, Fu KW, Law YW, Wong PW, Xu Y. Restricting the means of suicide by charcoal burning. Br J Psychiatri. 2010;196(3):241-2.

27. Miller M, Hemenway D. The relationship between firearms and suicide: a review of the literature. Aggress Violent Behav. 1999;4: 59-75.

28. Anglemyer A, Horvath T, Rutherford G. The accessibility of firearms and risk for suicide and homicide victimization among household members: a systematic review and meta-analysis. Ann Intern Med. 2014;160:101-13.

29. Siegel M, Rothman EF. Firearm ownership and suicide rates among US men and women, 1981-2013. Am J Public Health. 2016;106: 1312-22.

30. Fleischmann A, Bertolote JM, Wasserman D, De Leo D, Botega NJ, Da Silva D, et al. Effectiveness of brief intervention and contact for suicide attempters: a randomized controlled trial in five countries. Bull World Health Organ. 2008;86(9):703-9.

31. Mousavi SG, Amini M, Mahaki B, Bagherian-Sararoudi R. Effect of phone call versus face-to-face follow-up on recurrent suicide attempts prevention in individuals with a history of multiple suicide attempts. Adv Biomed Res. 2016;5:184.

32. Luxton DD, June JD, Comtois KA. Can postdischarge follow-up contacts prevent suicide and suicidal behavior? A review of the evidence. Crisis. 2013;34(1):32-41.

33. Rip JA, Privitera MR, West CP, Leiter R, Logio L, Shapiro J, et al. Well-being in graduate medical education: a call for action. Acad Med. 2017;92:914-7.

34. Karlsson P, Helgesson G, Titelman D, Sjöstrand M, Juth N. Skepticism towards the Swedish vision zero for suicide: interview with 12 psychiatrists. BMC Med Ethics. 2018;19:26. 